\title{
Multi-Domain Virtual Network Embedding with Coordinated Link Mapping
}

\author{
Shuopeng Li ${ }^{\text {}}$, Mohand Yazid Saidi, Ken Chen
}

L2TI, Institut Galiléee Université Paris 13 Sorbonne Paris Cité Villetaneuse, France

\begin{tabular}{l} 
A R T I C L E I N F O \\
\hline Article history: \\
Received: 03 April, 2017 \\
Accepted: 12 May, 2017 \\
Online: 01 June, 2017 \\
\hline Keywords : \\
Network Virtualization \\
Virtual network embedding \\
Multi-domain
\end{tabular}

\section{Introduction}

Network virtualization [1] is regarded as a solution to overcome some weakness of traditional network architecture. It makes easy to support various separated logical networks running over shared physical network. In virtualized network architecture, the service provider (SP) creates and manages virtual networks $(\mathrm{VN})$ for end users, while infrastructure provider (InP) deploys the substrate network (SN) equipment and offers the necessary physical resources.

An important step of network virtualization is to establish VNs above SNs. This is referred as virtual network embedding (VNE). The VNE problem aims to find a mapping from the $\mathrm{VN}$ to $\mathrm{SN}$ in a way that objectives (e.g. cost) are optimized and constraints (e.g. bandwidth) are satisfied.

Large networks in current Internet architecture are organized by autonomous system (AS). An AS is one or several physical networks controlled by a single authority. In this article, we use the vocabulary of "domain" to denote the whole substrate network under the control of a single InP.

As VNs are getting more and more deployed, VNs in multi-domain will be more and more considered by potential VN users. Establishment of multi-domain $\mathrm{VN}$ is more difficult than the one on single domain for at least two reasons:

- First, a single domain VNE problem is mainly solved by linear programming (LP). If we had a complete vision of all the domains, a multidomain VNE could be considered as a single domain VNE with a very large domain, so computationally harder to solve.

- More importantly, for various reasons (technical, commercial, etc.), the acquisition of full information in multi-domain is costly and often not possible. Only limited information is exchanged between InPs via protocols like BGP, so single domain approach cannot be re-used.

To address these challenges, multi-domain VNE

*Shuopeng Li, L2TI, Institut Galiléee Université Paris 13 Sorbonne Paris Cité Villetaneuse, France, li.shuopeng@univ-paris13.fr 
frameworks are developed. Usually, there is a VN decomposition step followed by local sub VN mapping in each InP. Some authors introduced a brokerlike additional actor, termed Virtual Network Provider (VNP) [2], between SP and InPs. The role of this VNP consists in assembling multi-domain information, decomposing $\mathrm{VN}$ and achieving the multi-domain VNE.

Existing solutions mainly focused on the decomposition of a multi-domain $\mathrm{VN}$ to each domain. One of the shortcomings in these frameworks is the lack of efficient link mapping method especially for the peering links which interconnect two domains.

In this paper, we propose an efficient framework of link mapping in multi-domain virtual network embedding context, which jointly consider the mapping of intra and peering links. In our approach, the peering links are mapped simultaneously along with intra domain links. Our approach is based on information usually disseminated by classical routing protocol (like BGP). Our simulation results prove that this solution results in better utilization of substrate resources.

The rest of this paper is organized as follows. Section 2 provides an overview of the related work. Section 3 presents our network model. Section 4 presents our multi-domain VNE solution. The evaluation results are shown in section 5 . Section 6 concludes this paper.

\section{Related Work}

\subsection{Single domain VNE}

The problem of single domain VNE is NP-hard [3] [4]. A basic off-line approach is proposed in [5], which performs the embedding in 2 stages (node then link mapping). The Multi Commodity Flow (MCF) is introduced in [6] to embed the virtual links The method in [7] takes into account the virtual links in node mapping stage. It privileges such node mapping that reduce the length of substrate link path.

Since 2-stage VNE solutions are lack of cooperation, some solutions mapping nodes and links in the same stage have been proposed. An approach based on subgraph isomorphism detection is presented in [8]. An other model in [9] applies the Markov Random Walk to rank nodes and then embeds links and nodes by using back-tracking strategy based on breadth-first search. In order to meet the change of requirements over time, dynamic VNE is proposed in [10] .

\subsection{Multi-domain information disclosure}

Because of politic and efficiency reasons, InPs can't disclose their complete information to others, so it is critical to make clear the information disclosure policy.

The proxy VNP could get peering links location and resource information but intra-domain links cannot be assumed to be available to VNP [11].
In [12], three types of resource information in each domain are provided to VNP:

- Node: its location, available capacity and unit price.

- Peering link: its vertices, available capacity and unit price.

- Intra-domain link: a length-based price for connecting any two nodes in its domain.

Based on the information disclosure policy above, we will describe our network model in the next section.

\subsection{Multi-domain VNE}

Multi-domain VNE framework can be decomposed into three major components [13]:

(i) partitioning the $\mathrm{VN}$ request into each $\mathrm{InP}$ via multi-domain node mapping method,

(ii) establishing inter-domain connection (peering links) between InPs using inter-domain paths,

(iii) embedding each sub $\mathrm{VN}$ request in each InP using intra-domain algorithm.

Based on multi-domain information model introduced in previous section, some centralized multi-domain VNE solutions are proposed in [14] 11][12] 15].

Many of them mainly focus on the first component. In [14], the authors introduce the cost of mapping a virtual node to a domain and the cost of mapping a link between two substrate nodes. Their node mapping algorithm optimizes the total embedding cost. The approach in [12] adopts the node mapping method [7] on a full-mesh topology which complies with partial information disclosure policy.

The second component is not very well explored compared to the first component. Existing solutions use simple policies to establish peering links. In [14], each peering link is considered as a single VPN (Virtual Private Network) connection. In [12], the flow of peering links is unsplittable between two domains, while the intra-domain sub virtual links are splittable. The peering link path is determined by Dijkstra's algorithm on VNP layer. Since VNP layer topology is not modified over time because of cost efficiency, Dijkstra's algorithm based peering links have always the same path. This phenomenon will result in difficulty of later intra-domain mapping. In [11], a virtual node is first mapped to substrate peering node to determine the peering link and the InP it belongs to. This approach is suitable for traffic matrix based VN [16] but not topology based VN.

Since establishing peering links is a part of link mapping, the chosen peering nodes will probably influence the intra-domain paths. We believe that there exist some inter-dependencies between the 2 nd and 
3rd components. To this end, we propose a framework which maps peering links jointly with intra-domain links in each InP.

\section{Network Model}

We adopt the usual substrate and virtual network model [7]. In addition, we describe VNP layer information based on existing multi-domain information model.

\subsection{Substrate Network}

A domain $\operatorname{In} P_{i}$ is modelled as an undirected graph $G_{i}^{S}\left(N_{i}^{S}, L_{i}^{S}\right)$, where $N_{i}^{S}$ is the set of substrate nodes in domain $i, L_{i}^{S}$ is the set of internal substrate links. Each substrate node $n_{i}^{S} \in N_{i}^{S}$ is associated with a CPU capacity $\operatorname{cpu}\left(n_{i}^{s}\right)$ and a geographic location $\operatorname{loc}\left(n_{i}^{s}\right)$. Each substrate link $l_{i}^{S} \in L_{i}^{S}$ is associated with a bandwidth capacity $b w\left(l_{i}^{s}\right)$.

Assuming that the substrate network covers $\mathrm{K}$ domains, there are some peering nodes (border nodes) which have peering links with other domains. The peering nodes set is denoted by $N_{i}^{S P}\left(N_{i}^{S P} \subset N_{i}^{S}\right)$. The peering links between $\operatorname{In} P_{i}\left(\right.$ i.e. $G_{i}^{S}$ ) and $\operatorname{In} P_{j}$ (i.e. $\left.G_{j}^{S}\right)$ is denoted by $L_{i j}^{S}$. We denote by $P_{i}^{S}=\cup_{j=1}^{K} L_{i j}^{S}$ the set of all of the peering links of $\operatorname{In} P_{i}$, and by $P^{S}$ with $P^{S}=\bigcup_{i=1}^{K} P_{i}^{S}=\bigcup_{(i, j) \in(1 \ldots K)^{2}} L_{i j}^{S}$ the set of all of the peering links. The complete substrate network $G^{S}\left(N^{S}, L^{S}\right)$ is thus obtained as follows: $N^{S}=\bigcup_{i=1}^{K} N_{i}^{S}$, $L^{S}=\left(\bigcup_{i=1}^{K} L_{i}^{S}\right) \cup P^{S}$.

\subsection{Virtual network}

The virtual network is also modelled as an undirected graph $G^{V}\left(N^{V}, L^{V}\right)$, where $N^{V}$ is the set of virtual nodes and $L^{V}$ is the set of virtual links. Each virtual node $n^{v} \in N^{V}$ is associated with a CPU capacity demand $c p u\left(n^{v}\right)$, a geographic location $\operatorname{loc}\left(n^{v}\right)$ and a distance constraint $\operatorname{dis}\left(n^{v}\right)$ specifying how far a virtual node $n^{v}$ can be placed from its $\operatorname{loc}\left(n^{v}\right)$. Each $l^{v} \in L^{V}$ is associated with a bandwidth demand $b w\left(l^{v}\right)$. In addition, each virtual network $G^{V}$ has a lifetime $t\left(G^{V}\right)$.

\subsection{VNP layer model}

VNP collects information provided by InPs. We assume that InPs provide exact information about their nodes, as well as the peering links. On the contrary, there is no exact information about the internal organisation of a domain. Similar to the existing solution [12], we assume that this information is given by $\mathrm{InP}$ for each couple of <node, peering node $>$, as if there was a pseudo direct link between these two nodes. Denote the set of these links by $L_{i}^{P}=\left\{l_{m n} / m \in N_{i}^{S}, n \in\right.$ $\left.N_{i}^{S P}\right\}, \operatorname{In} P_{i}$ provides to VNP the set of linking cost $C_{i}^{P}$ defined by

$$
C_{i}^{P}=\left\{C\left(l_{m n}\right) / m \in N_{i}^{S}, n \in N_{i}^{S P}\right\}
$$

where $C\left(l_{m n}\right)$ represents a cost (distance, bandwidth, etc.) characterizing the link $l_{m n}$. This kind of informa- tion is actually what a routing protocol (BGP) reports to other AS.

Thus, the $\mathrm{SN}$ of an $\operatorname{In} P_{i}$ is perceived by VNP as a graph $G_{i}^{P}=\left(N_{i}^{S}, L_{i}^{P}\right)$. In this way, the whole substrate network that VNP perceives, referred as $G^{P}$, is defined as follows:

$$
G^{P}=\left(\bigcup_{i} G_{i}^{P}\right) \bigcup P^{S}
$$

i.e. the perceived vision for each domain and the exact vision of the inter-domain connections. With $G^{P}$, VNP can establish a kind of complete topology covering all the domains for achieving $\mathrm{VN}$ decomposition and link mapping.

\section{Our proposition}

To solve VNE in the context of multi-domain, we propose a novel algorithm that maps jointly intra and peering links.

We propose to handle each $\mathrm{VN}$ request with a 2step process

- At the first step, VNP performs the node decomposition optimizing the node embedding.

- Subsequently, VNP performs a series of iterative downsizing VNE sub-solution, each of them optimizes both the intra and peering link mapping related to a domain.

The link mapping is determined, at each iteration, by the acting InP (called mapper). VNP is in charge of providing necessary information to the mapper. The generic work-flow of our algorithm is given by figure 1 . The details are explained as below.

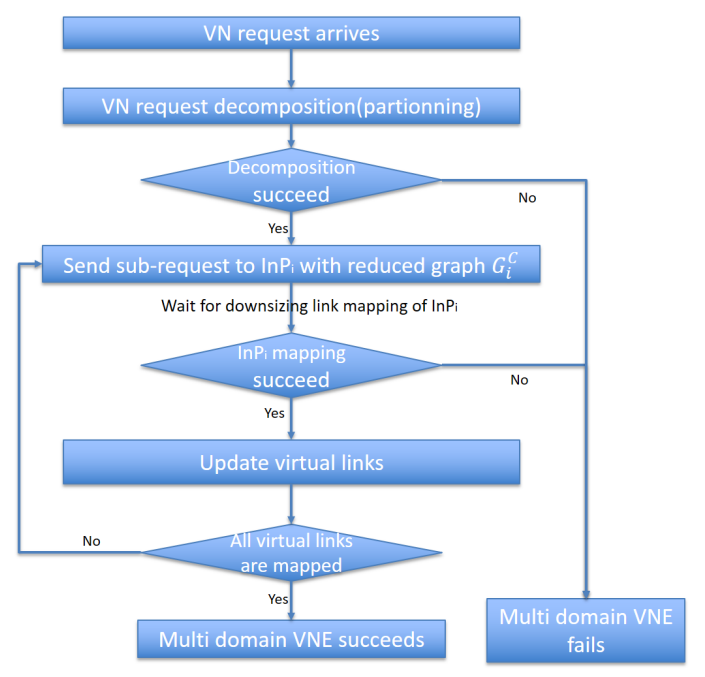

Figure 1: VNP workflow to embed a VN

\subsection{Decomposition}

Firstly, VNP decomposes the VN request with objective of minimizing the node mapping cost. In this stage, VNP associates each virtual node with a candidate set of substrate nodes that meet its $\operatorname{loc}\left(n^{v}\right)$. VNP is free to use any multi-domain VN partitioning 


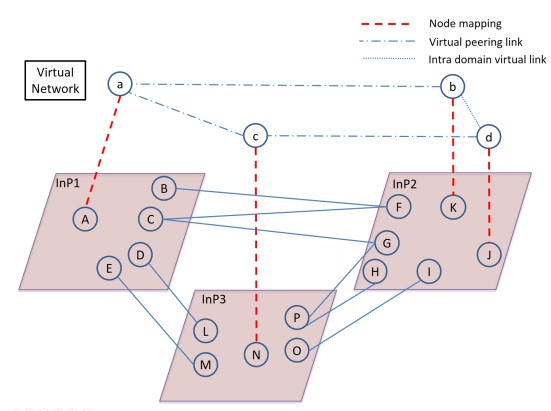

Figure 2: VN decomposition

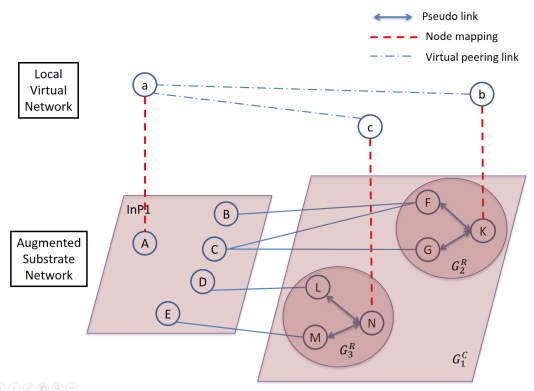

Figure 3: downsizing link mapping by $\operatorname{In} P_{1}$

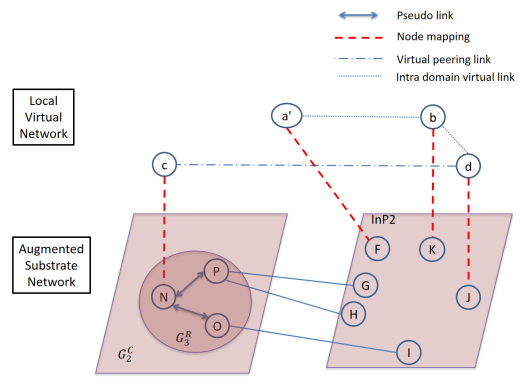

Figure 4: downsizing link mapping by $\operatorname{In} P_{2}$ method (e.g. [14] [12]). At the end of this stage, virtual nodes are embedded to different domains.

An example of $\mathrm{VN}$ decomposition is shown in figure 2. Three InPs are shown with their substrate nodes from A to P. They are connected via 2 or 3 peering links. Intra substrate links are not drawn. We suppose that a $\mathrm{VN}\{a, b, c, d\}$ arrives. The VN decomposition step tells us that $a, b, c$ and $d$ are mapped to substrate nodes $A, K, N$ and $J$, respectively. $\{a-b, a-c, c-d\}$ are virtual links which interconnect two different domains, while $\{b-d\}$ locates in only one domain.

\subsection{An iterative downsizing VNE ap- proach}

Here we give a detailed presentation of the kernel of our proposal, which is formally given in algorithm 1 .

\subsubsection{Rationale}

After VN decompostition step, since there is no domain who knows the complete information of any other one, embedding the virtual links which interconnect two different domains becomes an issue.

We notice that, VNP can build, for each $\operatorname{In} P_{i}$, a reduced vision (denoted by $G_{i}^{R}$ ) from $G_{i}^{P}$. This vision contains all the peering links/nodes, as well as the substrate nodes on which a virtual node is embedded. Formally, $G_{i}^{R}=\left(N_{i}^{R}, L_{i}^{R}\right)$ where

$$
N_{i}^{R}=N_{i}^{S P} \bigcup\left\{n_{i}^{S} \in N_{i}^{S} / \exists n^{v} \in N^{V}, M\left(n^{v}\right)=n_{i}^{S}\right\}
$$

i.e., $N_{i}^{R}$ is the union of all the substrate nodes supporting virtual nodes on domain $i$ and all of its peering nodes. In a similar way, we define $L_{i}^{R}$ as follows;

$$
L_{i}^{R}=\left\{l_{m n} \in L_{i}^{P} / n \in N_{i}^{R}, m \in N_{i}^{S P}\right\}
$$

i.e., $L_{i}^{R}$ is the subset of $L_{i}^{P}$ between $N_{i}^{R}$ and $N_{i}^{S P}$ containing only the links interconnecting a peering node and a node supporting a virtual node.

In order to achieve an efficient and pragmatic operation mode, we prefer that VNP plays its role of coordinator: It is VNP who decides which of the InP should have the privilege to map its peering links with others. It is also VNP who provides to the chosen InP (that we refer as mapper) the topology of the rest of the network according to its perception. In other words, the chosen InP (the mapper) extends its view to the rest of the network, by using the vision provided by VNP, the only one who has a kind of comprehensive view on all domains. In this way, the mapper obtains an augmented graph on which it will perform link mapping, including both its intra and peering links.

This process continues, domain after domain, until all of the virtual links are set. The selection criterion is the link utilization, the InP has most stringent link utilisation will be the first to map its peering links. The reason lies in that high link utilization denotes more constraints in the choice of path.

\subsubsection{Building of the augmented graph}

Let $\operatorname{In} P_{i}$ be the chosen mapper. Formally speaking, the vision of the other domains provided by VNP is $G_{i}^{C}=\bigcup_{j \neq i} G_{j}^{R}$, i.e. the reduced perceived vision of all the other domains. We only need to consider the case where all the domains are adjacent to the mapper. The case of a domain not adjacent to the mapper but to which the mapper has virtual links can be reduced to the adjacent case.

VNP communicates $G_{i}^{C}$ to the mapper $\left(\operatorname{In} P_{i}\right)$ so that the latter can creates an augmented graph $G_{i}^{A}$, defined as follows:

$$
G_{i}^{A}=G_{i}^{S} \cup P_{i}^{S} \cup G_{i}^{C}
$$

This topology covers all of the accessible domains and can be used as a substrate graph on which the mapper performs VNE.

\subsubsection{VN sub-request}

VNP asks the mapper to perform a partial VNE, which concerns only the virtual links related to the mapper. We refer this partial VNE as a sub-request $\left(L_{i}^{s u b v}\right)$. It is obtained from the current $\mathrm{VN}$ request by reducing it to virtual links related to the mapper.

\subsubsection{An MCF-based link mapping}

At this stage, the mapper gets an augmented vision of the whole substrate network, and a VNE sub request $\left(L_{i}^{s u b v}\right)$, both from VNP. We have thus a classical VNE problem that we solve with the multi commodity flow 
(MCF) based mapping algorithm (line 6 of algorithm 1 .

At the end of this step, $\operatorname{In} P_{i}$ pre-allocates resources on the intra and peering links related to it and sends to VNP a virtual link update notification.

Let us illustrate it by our example. Assume in figure 2 that $I n P_{1}$ is chosen as the 1 st mapper. VNP builds the VNP-level graph vision $G_{1}^{C}=G_{2}^{R} \cup G_{3}^{R}$ (see figure 3 with $G_{2}^{R}=(\{F, G, K\},\{F-K, G-K\})$ and $G_{3}^{R}$ $=(\{M, N, L\},\{M-N, L-N\})$. It builds also the subrequest $L_{1}^{s u b v}=\{a-b, a-c\}$, actually the virtual links $b$ - $d$ and $c$ - $d$ will be pruned since they haven't any extremity node supported by a substrate node in $\operatorname{In} P_{1}$. VNP then sends $G_{1}^{C}$ and $L_{1}^{s u b v}$ to $\operatorname{In} P_{1}$. The latter builds the augmented graph $G_{1}^{A}$ which includes $G_{1}^{S}$ (all the nodes and links in $\left.\operatorname{In} P_{1}\right)$, the peering links $(B-F, C-F$, $C-G, E-M)$, and $G_{C}^{1}$. In $P_{1}$ then applies the MCF-based algorithm to solve the embedding of $L_{1}^{s u b v}$ on $G_{1}^{A}$.

\subsection{Update and iteration}

After each sub-request, the mapper (say $\operatorname{In} P_{i}$ ) reports the results. In particular, it gives the results of the mapping of all of its inter-domain virtual links in the following manner.

Let $l^{v}(a, b)$ be the virtual link between a particular node $a \in \operatorname{In} P_{i}$ and a particular node $b \in \operatorname{In} P_{j}$, with $b w\left(l^{v}(a, b)\right)$ as the required bandwidth. The MCF algorithm will map $l^{v}(a, b)$ into one or several paths. Denote by $N^{F}$ the set of the peering nodes of $\operatorname{InP} P_{j}$ through which a fraction of $l^{v}(a, b)$ is mapped. After the link mapping of $\operatorname{In} P_{i}$, the set of virtual links $\left\{l^{v}(c, b)\right\}_{c \in N^{F}}$ is equivalent to the virtual link $l^{v}(a, b)$ with bandwidth demand:

$$
\sum_{c \in N^{F}} b w\left(l^{v}(c, b)=b w\left(l^{v}(a, b)\right)\right.
$$

It is to be pointed out that these links are totally inside in $P_{j}$ and they replace $l^{v}(a, b)$.

As the mapping of $\operatorname{In} P_{i}$ is achieved, it will no longer appear as domain in the subsequent problem which contains only the remaining domains. However, the achieved mapping concerns only the links related to $\operatorname{In} P_{i}$ (intra as well as peering), the part of inter-domain virtual links on the other domains still has to be mapped. Each of such inter-domain virtual link related to the mapper can be transformed into the above described equivalent set which will be added to each concerned domain. For the sake of reading simplicity, we prefer to give an informal description here, instead of a formal one, which would generate some more heavyly-indexed notations.

In this way, we obtain a new VNE problem with:

- at the SN level, the retreat of $\operatorname{In} P_{i}$ and all the peering links related to it;

- at the $\mathrm{VN}$ side, the retreat of all the virtual links internal to $\operatorname{In} P_{i}$ and the replacement of all the inter-domain virtual links related to $\operatorname{In} P_{i}$ by their equivalent set which are added to corresponding domain.
This allows us to execute iteratively the downsizing mapping described in $\S 4.2$ VNP repeats the process till its convergence which is certain, since the subset is reduced by at least one domain (the mapper) at each iteration.

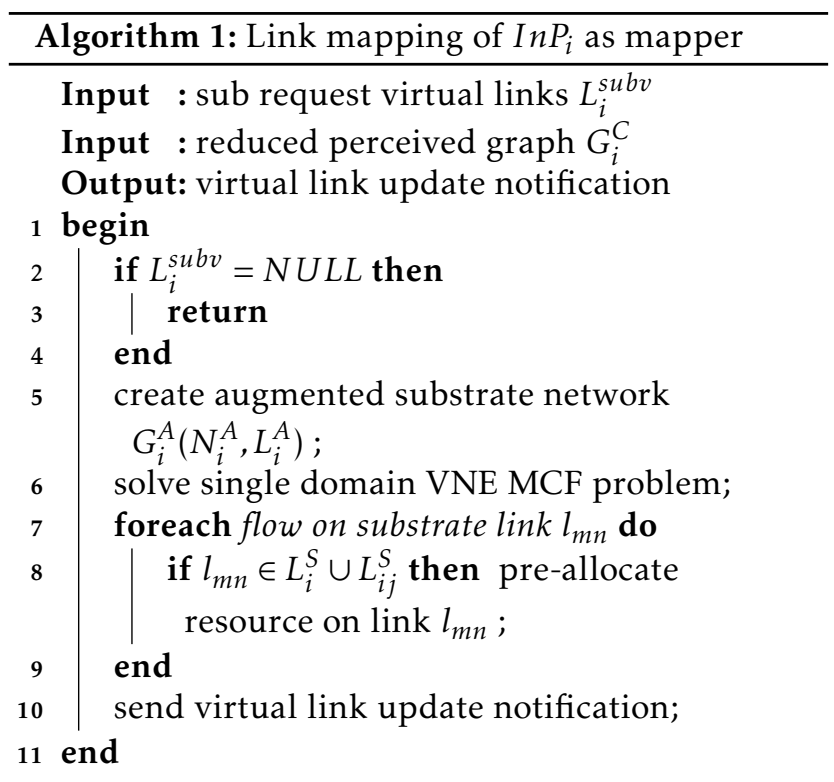

In the example of figure 2 and 3 , assume that $\operatorname{In} P_{1}$ has chosen link $F-K$ to map virtual link $a-b$. After sending its results to VNP, this latter deduces and creates a new virtual link $a^{\prime}-b$ with node mapping $a^{\prime}$ equal to $F$. This virtual link $a^{\prime}-b$ replaces virtual link $a-b$.

Now, the new problem (figure 4 contains only $\operatorname{In} P_{2}$ and $\operatorname{In} P_{3}$. Assuming that the $\operatorname{In} P_{2}$ is chosen as mapper, the same process continues and our problem is eventually reduced to a single domain which is the last step of our algorithm.

\subsection{Reject of virtual request}

The resources are definitively allocated only if all the computation on different domains succeed. A COMMIT message is then sent by VNP to InPs so as to validate the resource reservation. Should a mapper report a failure, a DEALLOC message would be sent by VNP, which stops the process (VNE failure) and allows each domain to deallocate pre-allocated resources.

\section{Reinforcement of our method}

As mentioned at the end of 4.2.1, we choose the link utilization criterion to determine mapping sequence. This choice simplifies the algorithm, but may fail to get the optimal solution. In this section, we propose a reinforcement of our algorithm, which waives the constraint of sequence selection.

Fundamentally, domains are peers. From a domain's point of view, there are actually 2 "domains" : a single domain (itself) and a outside domain (others). Using our downsizing algorithm, a domain tries its best to map its intra and peering virtual links, but how does the outside domain (others) map the remaining virtual links? We notice that after the first 
downsizing mapping, the problem is reduced to a multi-domain VNE on the outside domain (others) because the first mapper has mapped its own intra and peering virtual links. Following the downsizing logic, the problem will finally be reduced to a 2 domain VNE, on which a better solution can be easily found. Therefore, We first study the case of only 2 domains, and then we move to $\mathrm{K}$ domains VNE.

\subsection{Two domain basic method}

First, we consider the case of 2 domains. Assuming that the multi-domain VNE problem consists of 2 domains (denoted by $\operatorname{In} P_{1}$ and $\operatorname{In} P_{2}$ ), there are obviously 2 possible mapping sequences.

- First Solution $S_{1->2}: \operatorname{In} P_{1}$ starts up the mapping processus as mapper and then $I n P_{2}$ solves a single domain VNE.

- Second solution: $S_{2->1}: \operatorname{InP} P_{2}$ starts up the mapping processus as mapper and then $\operatorname{In} P_{1}$ solves a single domain VNE.

These two solutions are sent to VNP, which compares the embedding cost of these solutions. The final solution of the 2 domain basic multi-domain VNE (denoted by MDVNE(2)) is the better one among the 2 solutions above:

$$
S_{M D V N E(2)}=\min \left\{S_{1->2}, S_{2->1}\right\}
$$

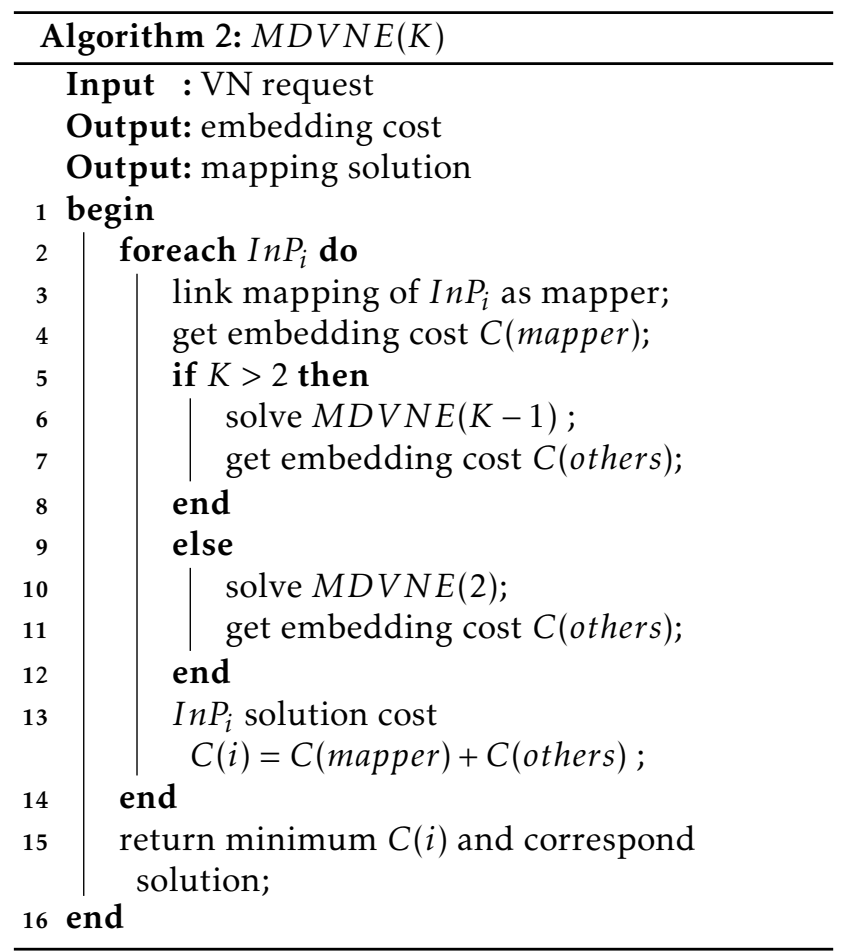

\subsection{Towards K domain solution}

From the 2 domain basic method, $\mathrm{K}$ domain multidomain VNE (denoted by $M D V N E(K)$ ) can be determined by a recursive algorithm. The detail of $M D V N E(K)$ is shown in Algorithm 2
The multi-domain is fundamentally divided into 2 elements, a mapper and the others. The former is mapped using our downsizing method (line 3 in Algorithm 2, and the later is reduced to a $\mathrm{K}-1$ domain problem (line 6 in Algorithm 2), until the basic 2 domain problem. The cost of the candidate is the sum of cost of the 2 elements (line 13 in Algorithm 2). The minimum cost candidate will be adopted as the mapping solution.

\section{Performance Evaluation}

We implemented a discrete event simulator to evaluate the performances of our method. The optimization problem is solved by IBM CPLEX library. Since we are basically interested by the link mapping, all the evaluated methods work with the same node decomposition by using the greedy algorithm of [6].

\subsection{Evaluation Environment}

The substrate networks are generated by GT-ITM tool [17]. 3 domains are generated. Each domain consists of 50 nodes and 100 links. The 3 domains are interconnected by 26 peering links. The CPU capacity of each node is chosen in $[50,150]$. The bandwidth capacity is selected in $[50,100]$ for intra links and in $[300,400]$ for peering links. The cost of the pseudo link between a border node and an intra node is chosen to be the inverse of the bandwidth capacity of the shortest path between these two nodes.

The virtual networks are also generated by GTITM tool. The virtual nodes of each VN follow a uniform distribution between 3 and 8 . The virtual nodes are interconnected with probability 0.4 . The CPU and bandwidth demands are uniformly chosen in $[0,20]$. The $\mathrm{VN}$ request arrival process is Poisson with arrival rate $\lambda \in(2 \ldots 6)$ requests per 100 time units. The life time of each $\mathrm{VN}$ follows an exponential distribution with an average of 1000 time units. Each simulation lasts for 20000 time units.

\subsection{Compared methods}

We compare the following 3 methods :

(i) lu-ciplm: Link utilization coordinated intra and peering link mapping. This is our first method.

(ii) $r$-ciplm: Reinforced coordinated intra and peering link mapping. This is our second method.

(iii) shen [12]: This approach computes separately intra and peering links. The latter is determined according to Dijkstra's algorithm.

We used the following metrics for comparison:

- VN request acceptance ratio: the ratio of the accepted $\mathrm{VN}$ request over the total arrived $\mathrm{VN}$ requests; 
- Average link utilization: the link utilization is the total allocated link resource over the total substrate resource. The allocated resource $\mathscr{U}$ is given by:

$$
\mathscr{U}=\sum_{l^{v} \in L^{V}} \sum_{l^{s} \in L^{S}} b w\left(l^{s}, l^{v}\right)
$$

where $b w\left(l^{s}, l^{v}\right)$ denotes the bandwidth committed on the substrate link $l^{s}$ to embed the virtual link $l^{v}$.

- Total revenue: The revenue of a $\mathrm{VN}$ as the weighted sum of bandwidth and CPU:

$$
\mathscr{R}=\beta \sum_{l^{v} \in L^{V}} b w\left(l^{v}\right)+\alpha \sum_{n^{v} \in N^{V}} c p u\left(n^{v}\right)
$$

where $\alpha$ (resp. $\beta$ ) is the unit revenue for cpu (resp. bandwidth). .

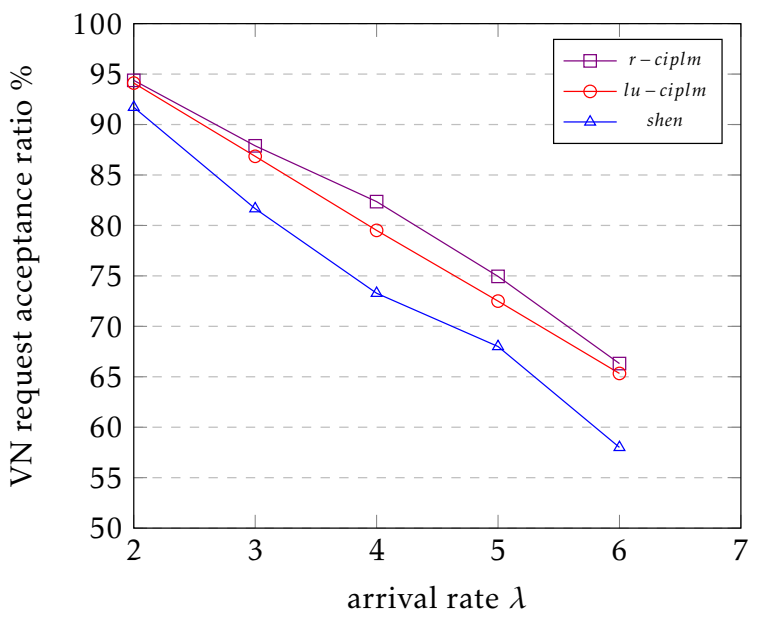

Figure 5: Acceptance ratio

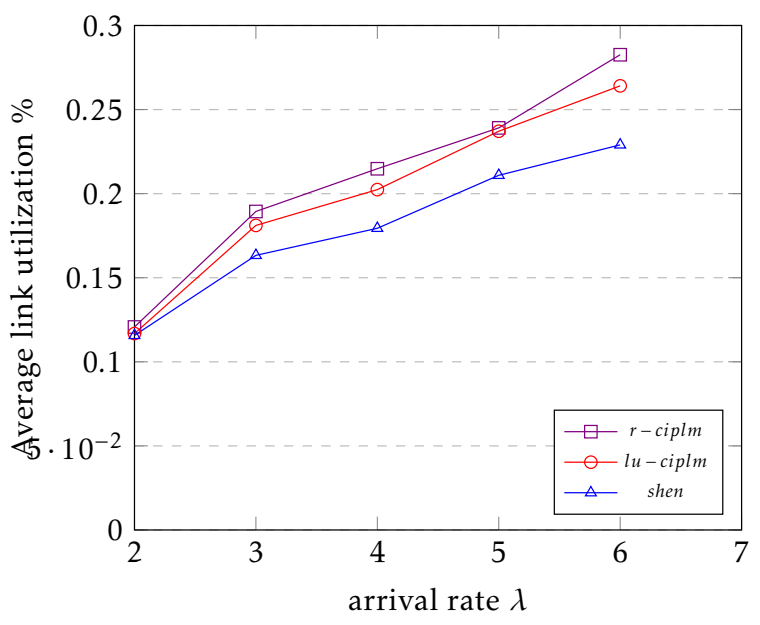

Figure 6: Average link utilization

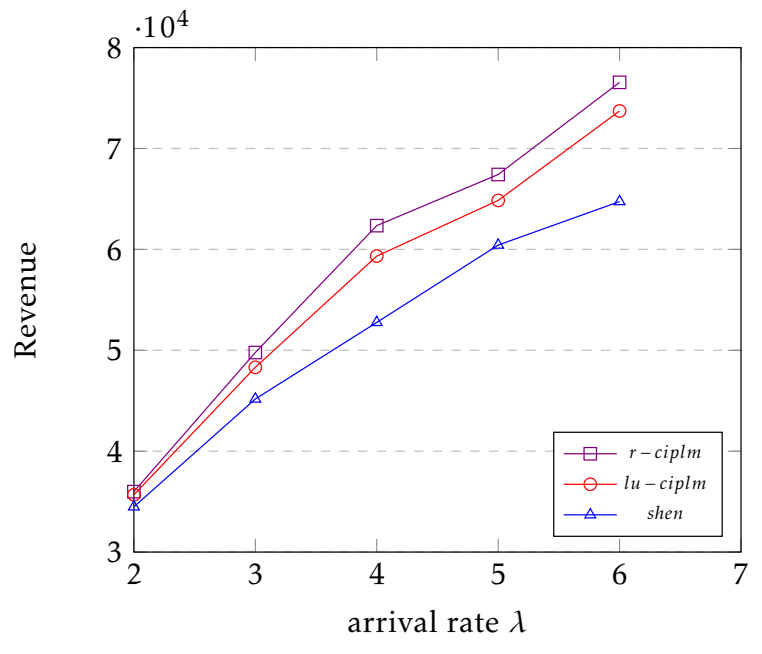

Figure 7: Total revenue

\subsection{Result analysis}

The simulation results are shown in Figure 5, 6 and 7. The $\mathrm{VN}$ request acceptance ratio is shown in Figure 5 The link utilization and revenue are shown in Figure 6 and Figure 7 , respectively. We got the following observations:

- $r$-cilpm is the best, followed by $l u-c i p l m$, and then shen over all the three metrics.

- The difference between $l u$-ciplm and shen is always significant.

- The difference between $r$-ciplm and $l u$-ciplm is not always significant.

To summarize:

- Our approaches are better than that of shen. Indeed, mapping jointly intra and peering links increases the efficiency. Our methods improve the performance. In these cases, traffic is splitted and sent to less loaded links, achieving in this way a better utilisation of the overall residual bandwidth.

- The out-performance of $r$-ciplm is small compare to $l u$-ciplm. $\quad r$-ciplm ensures a costefficient mapping solution for every VN. The peering links are mapped jointly with the right domain, which leads to a better resource allocation. However, the peering links in $l u-c i m p l m$ are sometimes not perfectly mapped because link utilization does not always give the best mapping sequence.

\section{Conclusion}

Network virtualization attracts more and more attention in future network architecture, since it allows the (dynamic) building of a network suited to endusers need, without modifying the underlay infrastructures. Part of them will be built over several infrastructures run by different operators. 
The virtual network embedding, which aims at establishing the optimal virtual networks on substrate networks, is a key issue in network virtualization. The fact of partial information makes the multidomain VNE quite different from the single-domain VNE and this problem remains a challenge. Some multi-domain VNE solutions have been proposed in literature. Most of them focus more on VN decomposition into sub VN requests for each domain, so that the single-domain VNE can be applied subsequently. Few attention has been paid on the mapping of peering (inter-domain) links.

In this paper, we propose a novel multi-domain VNE algorithm which aims to optimize the peering link mapping. For this, we introduce a coordinator (VNP, VN Provider). The latter has the privilege to get a comprehensive vision of all of the domains as well as the peering links. It performs VN decomposition, then coordinates the optimized mapping of both intra and peering links, domain after domain, in an iterative (and converging) manner. The optimization is achieved by applying the MCF algorithm on an augmented graph related to each domain. Simulation shows that our approach optimizes the substrate resource utilization compared to existing method. Besides, our method is easy to deploy in current Internet architecture.

\section{References}

[1] T. Anderson, L. Peterson, S. Shenker, and J. Turner, "Overcoming the internet impasse through virtualization," Computer, no. 4, pp. 34$41,2005$.

[2] G. Schaffrath, C. Werle, P. Papadimitriou, A. Feldmann, R. Bless, A. Greenhalgh, A. Wundsam, M. Kind, O. Maennel, and L. Mathy, "Network virtualization architecture: proposal and initial prototype," in Proceedings of the 1st ACM workshop on Virtualized infrastructure systems and architectures, pp. 63-72, ACM, 2009.

[3] A. Belbekkouche, M. Hasan, and A. Karmouch, "Resource discovery and allocation in network virtualization," Communications Surveys E Tutorials, IEEE, vol. 14, no. 4, pp. 1114-1128, 2012.

[4] A. Fischer, J. F. Botero, M. Till Beck, H. De Meer, and X. Hesselbach, "Virtual network embedding: A survey," Communications Surveys E Tutorials, IEEE, vol. 15, no. 4, pp. 1888-1906, 2013.

[5] Y. Zhu and M. H. Ammar, "Algorithms for assigning substrate network resources to virtual network components," in INFOCOM, vol. 1200, pp. 1-12, 2006.

[6] M. Yu, Y. Yi, J. Rexford, and M. Chiang, "Rethinking virtual network embedding: substrate sup- port for path splitting and migration," ACM SIGCOMM Computer Communication Review, vol. 38, no. 2, pp. 17-29, 2008.

[7] N. M. K. Chowdhury, M. R. Rahman, and R. Boutaba, "Virtual network embedding with coordinated node and link mapping," in INFOCOM, pp. 783-791, IEEE, 2009.

[8] J. Lischka and H. Karl, "A virtual network mapping algorithm based on subgraph isomorphism detection," in Proceedings of the 1st ACM workshop on Virtualized infrastructure systems and architectures, pp. 81-88, ACM, 2009.

[9] X. Cheng, S. Su, Z. Zhang, H. Wang, F. Yang, Y. Luo, and J. Wang, "Virtual network embedding through topology-aware node ranking," ACM SIGCOMM Computer Communication Review, vol. 41, no. 2, pp. 38-47, 2011.

[10] G. Sun, H. Yu, V. Anand, and L. Li, "A cost efficient framework and algorithm for embedding dynamic virtual network requests," Future Generation Computer Systems, vol. 29, no. 5, pp. 1265-1277, 2013.

[11] D. Dietrich, A. Rizk, and P. Papadimitriou, "Multi-domain virtual network embedding with limited information disclosure," in IFIP Networking Conference, 2013, pp. 1-9, IEEE, 2013.

[12] M. Shen, K. Xu, K. Yang, and H.-H. Chen, "Towards efficient virtual network embedding across multiple network domains," in Quality of Service (IWQoS), 2014 IEEE 22nd International Symposium of, pp. 61-70, IEEE, 2014.

[13] M. Chowdhury, F. Samuel, and R. Boutaba, "Polyvine: policy-based virtual network embedding across multiple domains," in Proceedings of the second ACM SIGCOMM workshop on Virtualized infrastructure systems and architectures, pp. 49-56, ACM, 2010.

[14] I. Houidi, W. Louati, W. B. Ameur, and D. Zeghlache, "Virtual network provisioning across multiple substrate networks," Computer Networks, vol. 55, no. 4, pp. 1011-1023, 2011.

[15] K. Guo, Y. Wang, X. Qiu, W. Li, and A. Xiao, "Particle swarm optimization based multi-domain virtual network embedding," in Integrated Network Management (IM), 2015 IFIP/IEEE International Symposium on, pp. 798-801, IEEE, 2015.

[16] C. Wang, S. Shanbhag, and T. Wolf, "Virtual network mapping with traffic matrices," in Communications (ICC), 2012 IEEE International Conference on, pp. 2717-2722, IEEE, 2012.

[17] GT-ITM http://www.cc.gatech.edu/projects/gtitm/. 